\title{
SIZING MANUFACTURING CELL MACHINES BASED ON THE SIMULATION AND AN EXPERT SYSTEM
}

\author{
Masmoudi, F. \\ Unit of Mechanics, Modeling and Production (U2MP), \\ Department of Mechanical Engineering, Engineering School of Sfax, \\ Route de Sokra Km 3.5 BP W, 3038 Sfax, Tunisia \\ E-Mail: faouzi.masmoudi@enis.rnu.tn
}

\begin{abstract}
This article developed a new method of the manufacturing cell sizing according to an approach based on the simulation and expert system. This method takes into consideration the stochastic aspect which governs the production system, the production scales of the product family to be treated, the products arrival law, the adopted scheduling and the used optimization criteria.

This study starts with the modeling of a manufacturing cell and the simulation of its functioning using the software "ARENA", which provides performance ratings. In a second stage, the expert system exploits these performance measures by a confrontation of the obtained results to the pre-defined objectives. This step permits to lead to a possible decision in order to size each of the machines to obtain a better manufacturing cell functioning. This process is repeated iteratively until the obtention of a cell having the performances which conform to the objectives already defined. The method represents a very useful and economical approach to predict the system size.

Finally, applications of the developed method for the sizing of manufacturing cell machines permitted to get satisfactory results.

(Received in June 2005, accepted in January 2006. This paper was with the author 4 months for 3 revisions.)
\end{abstract}

Key Words: Sizing, Manufacturing Cell, Simulation, Expert System, Performance Measures

\section{INTRODUCTION}

This article deals with the design of production cell by mean of sizing each machine used for product family process. There are many approaches to tackle this issue. They are classified into two main categories: the analytical method and simulation method.

The first category uses the mathematic modelling which allows to link parameters like the production needs and the abilities of different resources. Millers and Davis [1] proposed a methodology for the classification of this approach. The major disadvantage of this approach is its static and determinist character. Moreover, in this analytical approach, the dynamic aspect of the systems is not taken into consideration as well as the stochastic aspects due to certain factors like the control variation, the products arrival and the reliability of the resources. Similarly, the consistency with scheduling and piloting of the manufacturing systems is not assured. For this reason, the results obtained lack robustness. Besides, these methods provide a very weak general view of the manufacturing considered systems due to the study of resources problem selection independently of the other problems such as shop layout or material handling, etc.

Other analytical approaches developed mathematical and dynamic models [2, 3]. However, for the simulation and the study of a real case, the size of the problem to be solved becomes 
very important and consequently the quantity of information makes the mathematic formulation very complex.

The problem is also tackled by artificial intelligence techniques like the expert system. Indeed, the technology of the expert system was support to stand the mathematical models. As it was proposed by Kusiak [5], its role is to decide, in function of the available information, the size of the problem, the mathematical model and the resolution algorithm to use. The gaps mentioned by Miller and Davis [1] will always be present in spite of the intelligent aspect.

The second category of approaches is based on the simulation. Bullinger and Sauer [6] changed the number of production system resources to be optimised until obtaining a state judged satisfactory. Other authors performed simulation studies system design [7-9].

However, the simulation approaches are strongly associated with methods of trial and error. Therefore, the goal of these approaches is not the optimality but satisfaction results.

The main objective of this work is then to include the stochastic aspect which governs the arrival of products to the manufacturing cell as well as the parameters of the machine exploitation. Moreover, this study uses measures which characterise the performances of the manufacturing cell to be studied in order to make the best decisions during the sizing phase of the machines ability.

The rest of this paper is organised as follows: Section 2 describes the approach based on simulation and expert systems. Section 3 presents a case study and the obtained results. Finally, section 4 concludes the results and discussion.

\section{ADOPTED APPROACH}

The approach requires three main inputs:

- the necessary machines for the family of products to be treated,

- the transformation steps of the different products to be fabricated within the manufacturing cell. These supposed to be stable in time,

- the objectives to reach and their delimitating values.

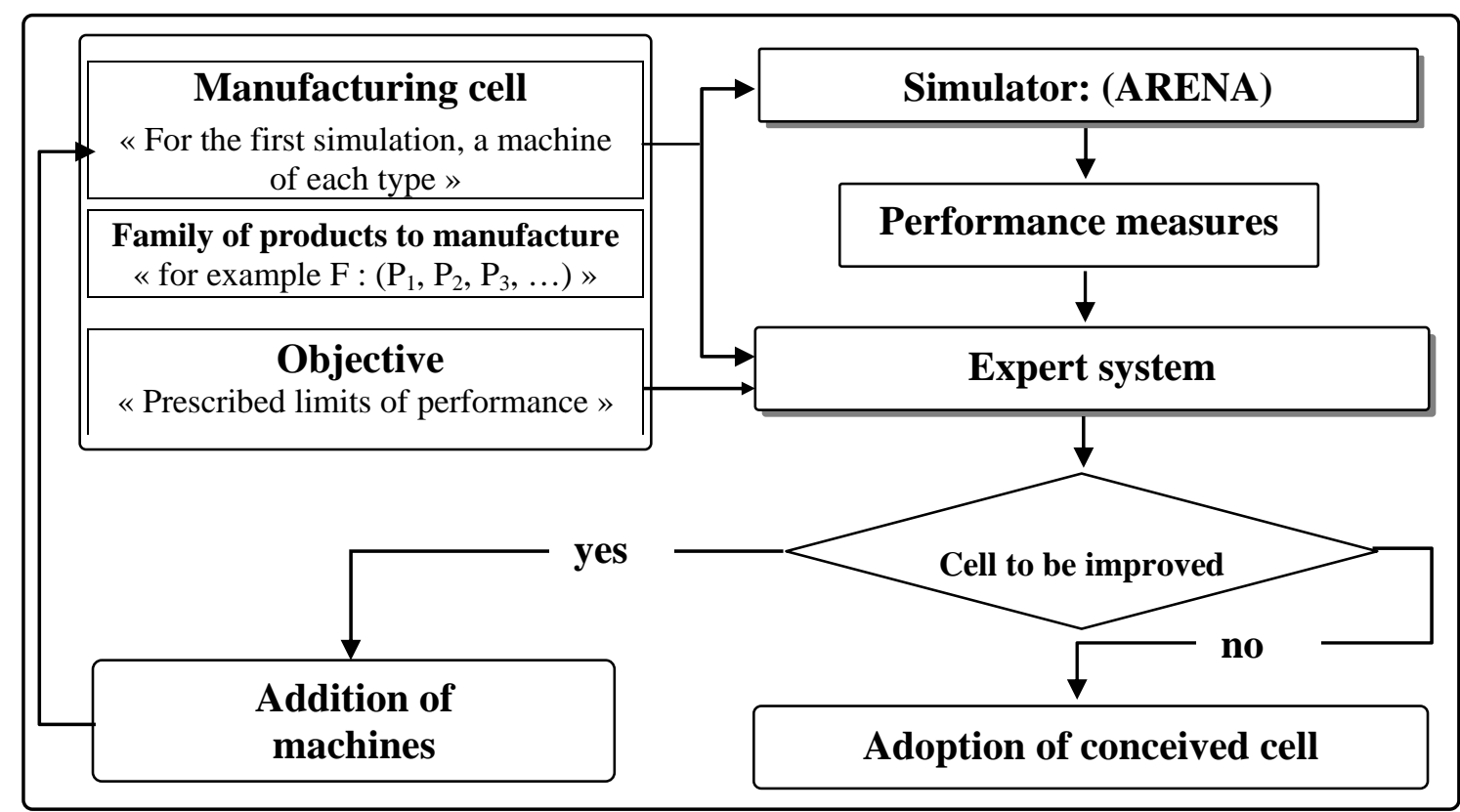

Figure 1: Optimisation cycle. 
The manufacturing cell is modelled by the simulator ARENA which simulates the processing of typical demand orders. The expected results will be the performance measures enabling the evaluation of the system with respect to the fixed objectives. These results, the maximum values of the performance measures as well as the number of each type of machine cell, are the inputs of the expert system.

Moreover, according to the analysis of the situation by the expert system with respect to the performance limits, two types of situations are possible:

- if the system performance, considering the current context, is non-improvable, then the procedure of optimization ends and the cell is to improved,

- if, the system needs to be improved and there is a lack or an excess of machines particular type, the role of the expert system, in this case, is to detect the problem and to recommend a modification in order to solve the problem considered as responsible for the failure to meet the objectives.

As a consequence of the second condition, a new cycle of optimisation is necessary after indicating the modifications according to the proposed decision.

The approach may be started from an initial system including a machine of each type necessary for the fabrication order. Then an iterative process will allow improving the performances in order to reach the objectives.

\subsection{Modelling of a manufacturing system by ARENA}

Fig. 2 shows a modelling of this system by the simulation software ARENA [10]. The entry of the product batches within the manufacturing cell is ensured by the module "CREATE".

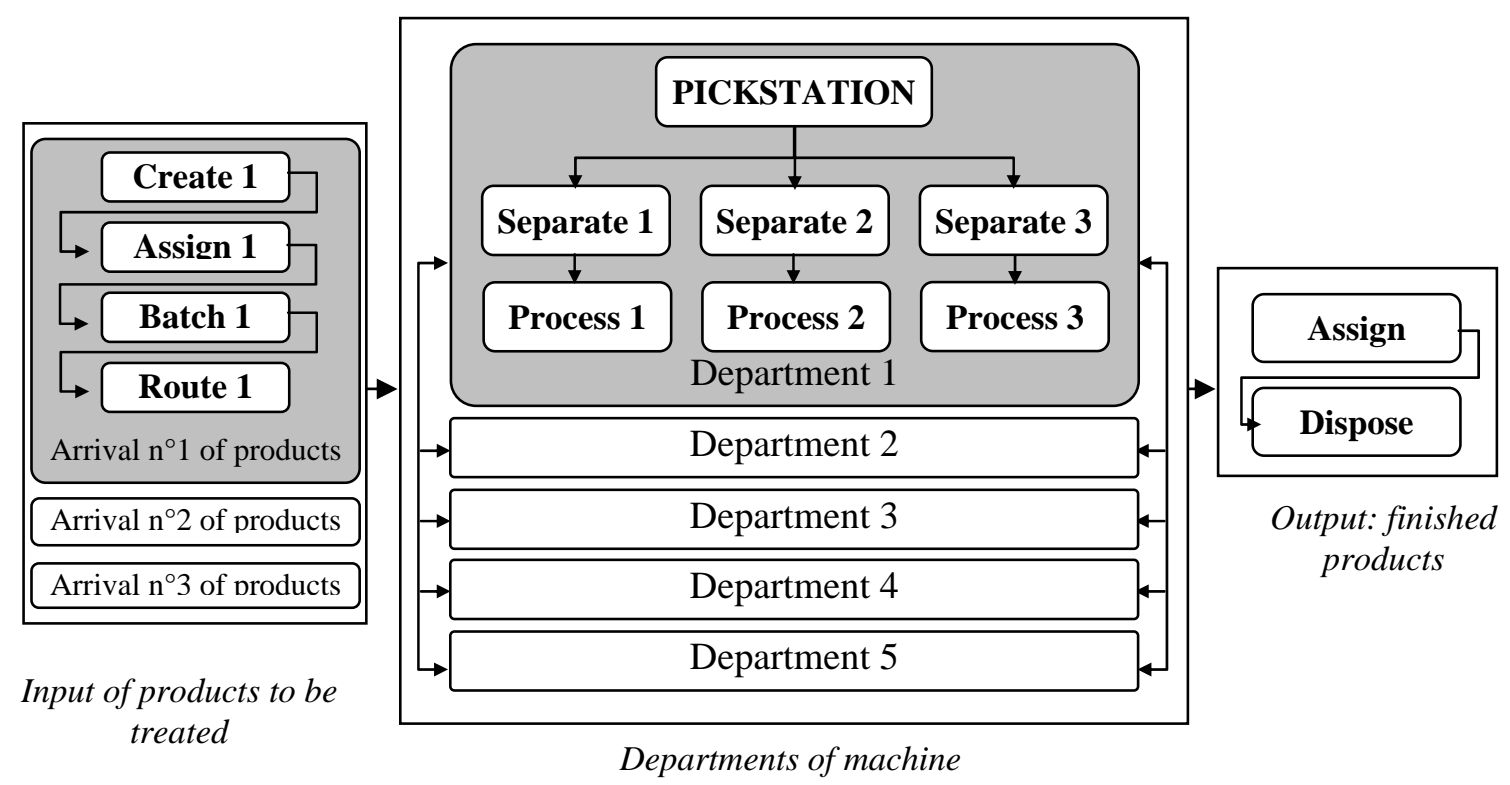

Figure 2: Modelling a manufacturing cell by ARENA.

Then, each batch is assigned a set of attributes via the module "ASSIGN". It is to be noted, at this level, that the batches are formed thanks to the module "BATCH", and they are moved by the module "ROUTE" towards the destination department.

Within the department, the choice of the candidate machine, modelled by the module "PROCESS" is ensured by the module "PICKSTATION". However, the transformation of the entities requires the split of the batches via the module "SEPARATE" first. Finally, and before the batches leave the manufacturing cell through the module "DISPOSE", the different 
variables considered as performance measures are calculated at the level of another modules “ASSIGN”.

\section{- Performance measures:}

For each objective of the firms, one or more criteria of performance may be defined. The coherence between the criteria of performance guarantees the overall performance of the system.

In this context of production at command in small sets, it would be interesting to consider the delivery deadline as the main objective. Thus, it would be necessary to minimize the delays with respect to the delivery deadlines causing penalties as well as the advances causing extra costs of bulwark. Nevertheless, this work hypothesizes that the advance cost is negligible and thus focuses only on minimizing the delay. For this reason, the suitable main performance measure would be thus the mean flow time (MFT) which should be minimized.

Moreover, a secondary performance measure would be necessary to detect the optimum number of the needed machines that can have a great influence on the MFT. Indeed, this second performance measure should allow us to determine the average waiting number $(W N)$. This index is the average number of batches queuing in front of a machine causing a bottleneck. However, in the presence of two machines having the same $W N$, but having different processing times, a third measure is deemed necessary. This performance measure is average waiting time $(W T)$.

On the other hand, the minimization of the batches (MFT) supposes the addition of machines. However, every machine has a purchase cost and its addition implies an additional investment. Consequently, the addition of a machine should be justifiable and relevant. In this context, it is necessary to set for each type of machine a minimal limit of use taking in account its technical and financial features. The rate of utilisation $(R U)$ is defined as the ratio between the occupation time of the machine and the operations total time.

\section{- Formulation of performance measures:}

The definite parameters, here under, enter in the formulation of performance measures.

$d$ : type of the machine in the manufacturing cell, each type has a number $d=1,2, \ldots, D$

$P$ : number of the products types, a type of product is labelled $p=1,2, \ldots, P$

$B$ : number of batches, each one is labelled by $b=1,2, \ldots, B$

$S d$ : number of machines of type $(d)$, each one will be labelled by $s=1,2, \ldots, S d$

TT: total time of the necessary operations to finish the order to manufacture.

Measures of performance permitting the analysis of production cells by the expert system are defined as follows:

\section{- Mean flow time}

The products being launched by batches, each batch $(b)$ remains in the manufacturing cell during a certain period of time called Flow Time $F T^{b}$. The MFT in the manufacturing cell is obtained by averaging the flow time of the batches.

$$
M F T=\frac{1}{B} \sum_{b=1}^{B} F T^{b}
$$


- Number waiting

With the arrival of batch $(b)$ to the machines of type $(d)$, two cases are possible:

- The machine $(s)$ is available and the batch $(b)$ is immediately treated.

- The machine $(s)$ is not available and the batch $(b)$ must queue up before being treated.

The average number of batches queuing up for a period of time in front of the machine number (s) of type $(d)$ is given by $N W_{d s}$.

Consequently, the average number of batches queuing up in front of the machine of type $(d)$ is given by:

$$
N W_{d}=\frac{1}{S_{d}} \sum_{s=1}^{S_{d}} N W_{d s} \quad \text { with } d=1, \ldots, D
$$

\section{- Waiting time}

The batches which were queuing up in front of the machine (s) of type $(d)$, have in average a waiting time given by $W T_{d s}$.

Consequently, the waiting time of the batches queuing up in front of the machines of type (d) is:

$$
W T_{d}=\frac{1}{S_{d}} \sum_{s=1}^{S_{d}} W T_{d s} \text { with } d=1, \ldots, D
$$

\section{- Rate of utilisation $\left(R U S_{d s}\right)$}

The utilisation rate of machine $(s)$ of type $(d)$ is the ratio of the machine occupation time and the total time of operations.

$$
R U S_{d s}=\frac{P T_{d s}+S T_{d s}}{T P T} \quad \text { with } d=1, \ldots, D \text { and } s=1,2, \ldots, S_{d}
$$

Where,

$P T_{d s}$ is the total time of operation on the machine $(s)$ of type $(d)$ found out by the addition of all the processing times of the different batches $P T^{b}{ }_{d s}$.

$$
P T_{d s}=\sum_{b=1}^{B} P T_{d s}^{b} \quad \text { with } d=1,2, \ldots, D \text { and } s=1,2, \ldots, S_{d}
$$

Where,

$S T_{d s}$ is the total setup time of the machine (s) of type (d) given by the addition of $S T_{d s}^{b}$ of all the setup times of the machine $(s)$ of type $(d)$ for treatment of batch $(b)$.

$$
S T_{d s}=\sum_{b=1}^{B} S T_{d s}^{b} \quad \text { with } d=1, \ldots, D \text { and } s=1,2, \ldots, S_{d}
$$

Finally, the average rate of machines utilisation of type $(d)$ is:

$$
R U_{d}=\frac{1}{S_{d}} \sum_{s=1}^{S_{d}} R U S_{d s} \quad \text { with } d=1, \ldots, D
$$




\subsection{Expert system}

\section{- Structure of the system expert}

The expert system represents a tool for making decision. Since the primary objective of this study is to improve the throughput of a manufacturing cell, a simulation analysis assisted by the expert system decision process is carried out. A fact list that supports all the required information for decision making before executing the system expert needs to be developed first. The structure of the expert system given in Fig. 3 is composed of two bases: basis of objects and basis of rules. This structure is expressed in terms of inference rules, such us: if (condition) then (action) grouped in several packs.

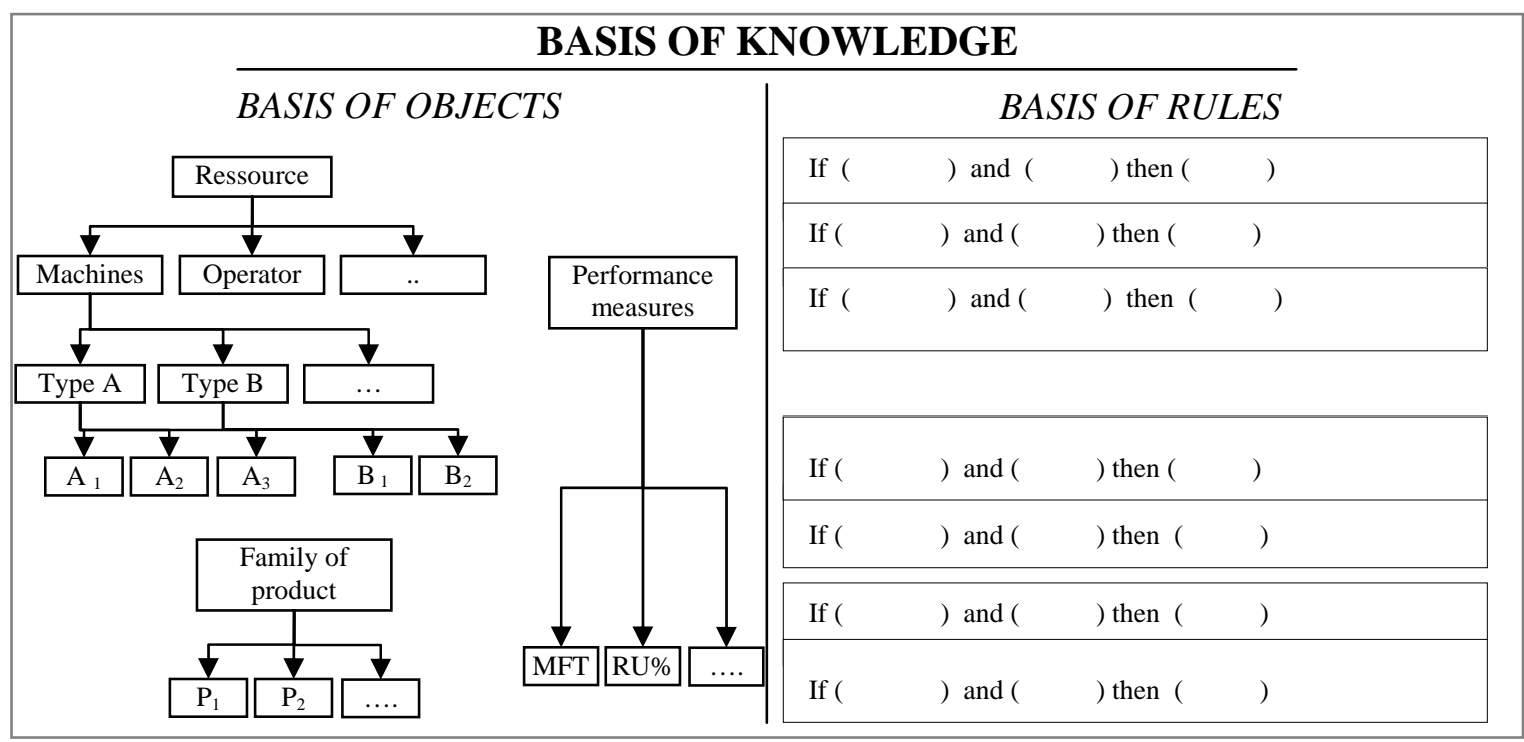

Figure 3: Structure of the expert system.

\section{- Working procedure of the expert system}

First, the current configuration of the manufacturing cell is used as the input data to build a simulation base model. The simulation run of the base model will produce the current cell performance, such as $M F T, N W, T W$ and $R U$ which will be the input of the expert system. If the performance target is not achieved, the expert system will recommend how to modify the simulation model by varying the number of machines. The iterations continue until the performance target of the cell is met (MFTmin and $R U_{d}$ limit).

Once the expert system has made the recommendations, the simulation model is adjusted accordingly and rerun. The simulation component and expert system component are cooperating with each other until the target throughput is achieved.

In order to provide decision support for identifying bottleneck machines, the procedure of the expert system devises four phases which are shown in Fig. 4. Theses phases are as follows:

Phase 1: Collection of data; this phase includes manufacturing cell configuration, simulation output and performance criteria objective. In this research, the performance criteria consist of mean flow time (MFT) and the limits authorized of the rate of machines utilisation $\left(\mathrm{RU}_{\mathrm{d}}\right.$ limit).

Phase 2: Analysis of data; this phase consists in analysing the data collected from the simulation phase. The result of this phase is to identify the bottleneck machines one by one.

Phase 3: Suggesting recommendations, "searching lack and or excess of machines"; this phase consists to identify the bottleneck machine according to the performance measures. 
Once these parameters are detected, it arranges by order of importance, the corresponding recommendations according to two lists: the list of lack of machines and that of excess of machines for each type.

If the state of the system is not satisfactory, therefore this indicates that the current recommendation carried did not lead to the improvement of the manufacturing cell. Consequently, this recommendation is not retained and the expert system recovers the remainder of the unexplored recommendations of the previous cycle and it checks their feasibility.

Phase 4: Checking the feasibility of the proposed recommendation, this last phase consists to apply the procedure which given in Fig. 4. For example the withdrawal of a single machine in a unit, would be excluded from the list of recommendations.

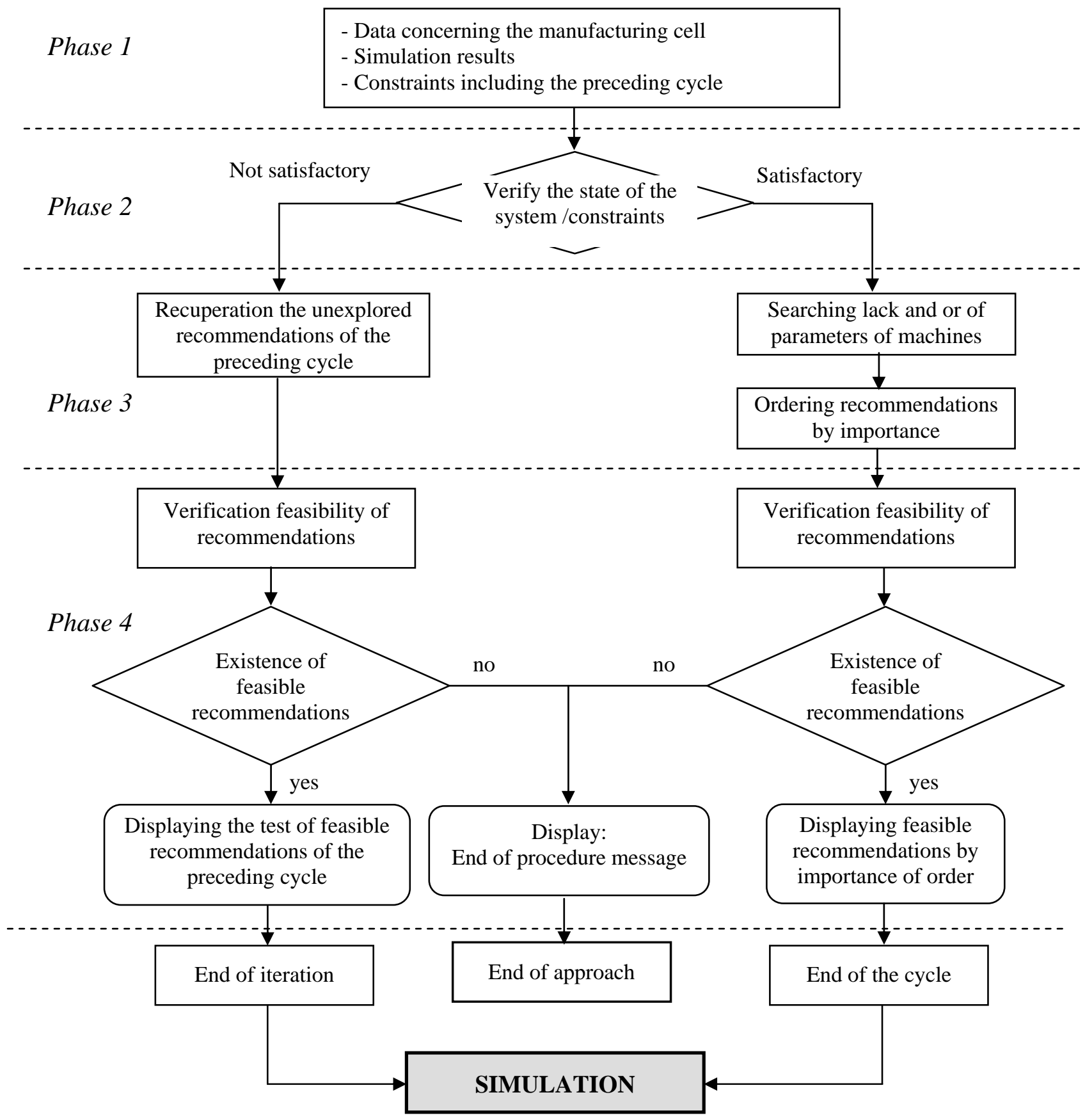

Figure 4: Resolution procedure. 
Therefore, two possibilities are considered. The first is that there is no longer any feasible recommendation suggested by the expert system at the end of the previous cycle. This implies the end of the procedure and the manufacturing cell cannot be improved. The second is that there are one or more feasible recommendations of the previous cycle. The expert system displays them in order of importance and indicates the end of the iteration. In this case, one iteration is one attempt of adoption of one of the given cycle recommendation. Each iteration may lead to the end of the cycle if the last recommendation is retained.

The expert system verifies the global performance measures. Following this verification, two cases are possible. The state of the system is improved or not. If the state is either improved, the expert system should detect the parameters linked to lack and/or excess of each type of machine. The absence of any recommendation implies the end of the procedure and approach.

\section{AN ILLUSTRATIVE EXEMPLE}

In order to avoid any bias and to give the maximum of generality to this application, the following characteristic of the manufacturing system and the fabrication order were retained:

- the family of products comprises three different types of products (Table1),

- for one type of product, the transformation time differs from one machine to another,

- the handling of the pieces between workstations is done by batches of the same size of the product order,

- the setup time is always lower than the processing time of a piece and for each of the three types of productions,

- the unit contains five machines, one of each type,

- for the five type $\mathrm{M}_{1}, \mathrm{M}_{2}, \mathrm{M}_{3}, \mathrm{M}_{4}$, and $\mathrm{M}_{5}$, the maximum utilization rate is $100 \%$ and the minimum is $20 \%$,

- the arrival of the batches is generated by a Poisson law, and the preparation of workstations follows a triangular low of distribution [11],

- the management policy of the queues is first arrived first served.

Table I: Fabrication order specification.

\begin{tabular}{|c|c|c|c|c|}
\hline $\begin{array}{l}\text { Family of } \\
\text { products }\end{array}$ & Routing & Machines & $\begin{array}{c}\text { Processing } \\
\text { time }\end{array}$ & Setup time \\
\hline \multirow{3}{*}{$\mathbf{P}_{1}$} & 1 & $\mathrm{M}_{1}$ & 30 & Trian. $(55,60,65)$ \\
\hline & 2 & $\mathrm{M}_{4}$ & 30 & Trian. $(55,60,65)$ \\
\hline & 3 & $\mathrm{M}_{5}$ & 30 & Trian. $(55,60,65)$ \\
\hline \multirow{3}{*}{$\mathbf{P}_{2}$} & 1 & $\mathrm{M}_{4}$ & 90 & Trian. $(40,45,55)$ \\
\hline & 2 & $\mathrm{M}_{5}$ & 90 & Trian. $(40,45,55)$ \\
\hline & 3 & $\mathrm{M}_{3}$ & 90 & Trian. $(40,45,55)$ \\
\hline \multirow{3}{*}{$\mathbf{P}_{3}$} & 1 & $\mathrm{M}_{2}$ & 60 & Trian. $(50,60,70)$ \\
\hline & 2 & $\mathrm{M}_{3}$ & 60 & Trian. $(50,60,70)$ \\
\hline & 3 & $\mathrm{M}_{1}$ & 60 & Trian. $(50,60,70)$ \\
\hline
\end{tabular}


Once the limits of the performance are fixed, the specification of the product order is definite and the initial system modelled, the cycle zero may be launched. The first phase of this cycle is the simulation of the realisation of the order within a cell. The result of this phase, realised with the help of the simulator ARENA, is shown in table II.

Table II: Results of the simulation of cycle zero.

\begin{tabular}{|c|c|c|c|}
\hline $\begin{array}{c}\text { Machine } \\
\text { (numbers) }\end{array}$ & $\begin{array}{c}\text { NW } \\
\text { (batches) }\end{array}$ & $\begin{array}{c}\text { WT } \\
\text { (min) }\end{array}$ & $\begin{array}{c}\text { RU } \\
\text { (\%) }\end{array}$ \\
\hline $\mathbf{M}_{1}=1$ & 3118 & 27180 & 100 \\
\hline $\mathbf{M}_{2}=1$ & 1358 & 15851 & 100 \\
\hline $\mathbf{M}_{3}=1$ & 3081 & 28817 & 100 \\
\hline $\mathbf{M}_{4}=1$ & 3211 & 28387 & 100 \\
\hline $\mathbf{M}_{\mathbf{5}}=1$ & 0.2 & 3 & 100 \\
\hline \multicolumn{4}{|c}{ MFT $=47087$ (min) } \\
\hline
\end{tabular}

Table III: Modifications applied in the various cycles of optimisation.

\begin{tabular}{|c|c|c|c|c|c|c|c|c|}
\hline \multirow{2}{*}{ Cycle } & \multirow{2}{*}{ Iteration } & \multicolumn{5}{|c|}{ Machine numbers } & \multirow{2}{*}{$\begin{array}{l}\text { MFT } \\
\text { (min) }\end{array}$} & \multirow{2}{*}{$\begin{array}{l}\text { Recommendations by } \\
\text { order of priority }\end{array}$} \\
\hline & & M1 & M2 & M3 & M4 & M5 & & \\
\hline 0 & - & 1 & 1 & 1 & 1 & 1 & 4 & +M4 +M1 +M3 +M2 +M5 \\
\hline 1 & 1 & 1 & 1 & 1 & 2 & 1 & 46 & +M5 +M3 +M1 +M2 +M4 \\
\hline 2 & 1 & 1 & 1 & 1 & 2 & 2 & 33 & +M3 +M1 +M2 +M4 +M5 \\
\hline 3 & 1 & 1 & 1 & 2 & 2 & 2 & 21 & +M1 +M2 +M3 +M4 +M5 \\
\hline 4 & 1 & 2 & 1 & 2 & 2 & 2 & 5780 & +M2 +M3 +M5 +M4 +M1 \\
\hline 5 & 1 & 2 & 2 & 2 & 2 & 2 & 8868 & $+\mathbf{M} 3+\mathrm{M} 5$ +M4 +M1 \\
\hline 5 & 2 & 2 & 1 & 3 & 2 & 2 & 5157 & +M2 +M5 +M4 +M1+M3 \\
\hline 6 & 1 & 2 & 2 & 3 & 2 & 2 & 6128 & +M5 +M4 +M1+M3 \\
\hline 6 & 2 & 2 & 1 & 3 & 2 & 3 & 5140 & +M2 +M4 +M1+M3 +M5 \\
\hline 7 & 1 & 2 & 2 & 3 & 2 & 3 & 6094 & +M4 +M1+M3 +M5 \\
\hline 7 & 2 & 2 & 1 & 3 & 3 & 3 & 5127 & +M2 +M1 +M4 +M5 +M3 \\
\hline 8 & 1 & 2 & 2 & 3 & 3 & 3 & 6036 & +M1 +M4 +M5 +M3 \\
\hline 8 & 2 & 3 & 1 & 3 & 3 & 3 & 5126 & +M2 +M3 +M5 +M4 +M1 \\
\hline 9 & 1 & 3 & 2 & 3 & 3 & 3 & 51,68 & +M3 +M1 +M4 +M5 -M2 \\
\hline 10 & 1 & 3 & 2 & 4 & 3 & 3 & 50,73 & +M1 +M5 +M4 -M3 -M2 \\
\hline 11 & 1 & 4 & 2 & 4 & 3 & 3 & 50,43 & +M5 +M4 -M1 -M3 -M2 \\
\hline 12 & 1 & 4 & 2 & 4 & 3 & 4 & 50,43 & +M4 -M5 -M1 -M3 -M2 \\
\hline 13 & 1 & 4 & 2 & 4 & 4 & 4 & 50,37 & -M5 -M4-M1-M3-M2 \\
\hline 14 & 1 & 4 & 2 & 4 & 4 & 3 & 50,42 & $\begin{array}{l}+\mathrm{M} 5 \text {-M4 -M1 -M3-M2 } \\
\rightarrow \text { End of procedure }\end{array}$ \\
\hline
\end{tabular}

The second phase of this cycle zero: the analysis of the performances of the manufacturing system was carried out by the expert system. The latter checked that the state of the system in comparison with the constraints is generally satisfactory. It then moves to 
search problems of lack or excess of resources in each department. According to Table I, the most overloaded department among the five is first department since it has the greatest average number of batches waiting. Then, the suggested recommendation by the expert system was for this cycle: "the addition of a machine M1". In total, fourteen cycles and nineteen simulations were necessary to reach a non improvable performance of the manufacturing system.

The different modifications applied to the different cycles of optimisation of this application are summarised in Table III.

Since each department in the initial system contains only one machine, and taking into consideration the given modifications in Table III, the system finally obtained may be given by Table IV.

Table IV: Number of machines of the final system.

\begin{tabular}{|c|c|}
\hline Department & Number of machines \\
\hline M 1 & 4 \\
\hline M 2 & 2 \\
\hline M 3 & 4 \\
\hline M 4 & 4 \\
\hline M 5 & 3 \\
\hline
\end{tabular}

This system presents improved performances. Fig. 5 presents the evolution of the mean flow time (MFT) whose minimisation was our objective.

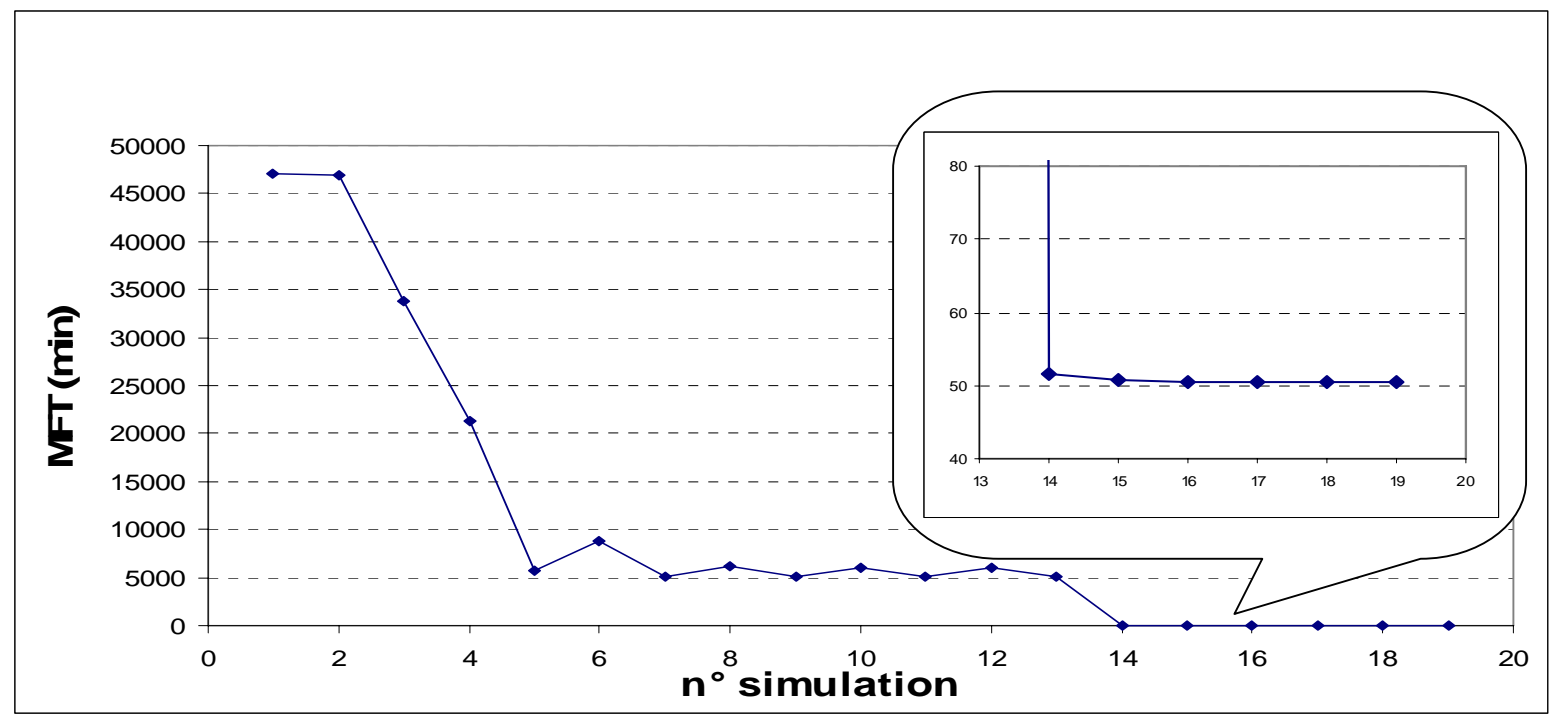

Figure 5: Evolution of MFT according to the number of simulation.

Moreover, a study of the initial solution choice influence on the final manufacturing system. The method was applied to the same studied application starting from another initial manufacturing system. The final solution obtained is the same found previously. The result in terms of the mean flow time is equally the some. Thus, the choice of the initial manufacturing system does not seem to have an influence on the final solution.

A complementary simulation also shows that the choice of the utilization rate limits may influence the final solutions of the method. The user has to choose between two possibilities: 
- to invest and to buy more machines even if they are not very well used and this to improve the mean flow time,

- to take the liberty of not having a minimum mean flow time and be satisfied with on acceptable solution. On the other hand, the profit will be economic at the level of investment.

\section{CONCLUSION}

This study attempted to develop a method that incorporates the stochastic aspect and uses performance measures better adapted to the production of small and medium series. It is expected to yield satisfactory results in applications where the products are well defined. The study concentrated mainly on sizing manufacturing cell machines. In the study, output of the number waiting, total waiting time, utilisation of resources and mean flow time were considered in the evaluation of the system. This sizing must respect the existing constraints and reach the pre-set objectives. The results would enable the company to gain a greater understanding of the behaviour of the systems and would permit to choose the most suitable design for the new plant. With the assistance of expert system, a set of systematic procedures can be defined for the user.

The use of simulation techniques can help to propose and evaluate modifications so as to improve the logistics performance. Besides, it should be clearly understood that further work on this system will yield an optimisation of the objectives in function of new performance measures.

\section{ACKNOWLEDGEMENT}

The author would like to thank Mr. A. Hajji from the Engineering School of Sfax for his help with English.

\section{REFERENCES}

[1] Miller, D. M; Davis, R. P. (1977). The machine requirements problem, International Journal of Operations Research, Vol. 15, 219

[2] Miller, D. M.; Davis, R. P. (1978). A dynamic resource allocation model for a machine requirements problem, AIIE Transactions, Vol. 10, No. 3, 237-243

[3] Sarin, S. C.; Chen, C. S. (1986). A model for manufacturing system selection: Methods and Studies, A Kusiak (Ed.), Amsterdam, 99-112

[4] Kusiak, A. (1987). Artificial intelligence and operation research in flexible manufacturing systems, Information processing and operation research, Vol. 25, No 1.

[5] Kusiak, A. (1990). Intelligent manufacturing systems, Prentice-Hall, New York

[6] Bullinger, H. J.; Sauer, H. (1987). Planning a flexible assembly system supported by simulation, International Journal of Production Research, Vol. 25, No. 11, 1625-1634

[7] Ashayeri, J.; Gelders, L. (1988). Production planning evaluation of PCB assembly through simulation, Proceedings of fourth international conference Simulation in manufacturing, Leuven, Belgium, 63-70

[8] Doerr, K.; Magazine, M. J. (2000). Design, coordination and control of hybrid factories. International Journal of Operations and Production Management, Vol. 20, No. 1, 85-102

[9] Peng, Q.; Skinner, G. E.; Mason, S. J. (2001). Sizing a pilot production line using simulation, Proceedings of the 2001 Winter Simulation Conference

[10] Arena Standard User's Guide, Doc ID ARENAS-UM001C-EN-P, 2002, Rockwell Software Inc.

[11] Newman, W. R.; Jo Maffei, M. (1999). Managing the job shop: simulating the effects of flexibility, order mechanisms and sequencing rules, Integrated Manufacturing Systems, 226-275 